Licença CC BY Artigo distribuído sob os termos Creative Commons, permite uso e distribuição irrestrita em qualquer meio desde que o autor credite a fonte original

\section{ADOPCIÓN DE YIELD MANAGEMENT EN HOTELERÍA: ETAPA PRE-COVID-19 Y EXPECTATIVAS EN EL CONTEXTO ACTUAL DE PANDEMIA}

\author{
ADOÇÃO DE YIELD MANAGEMENT EM HOTÉIS: ESTÁGIO PRE- \\ COVID-19 E EXPECTATIVAS NO ATUAL CONTEXTO PANDÊMICO \\ ADOPTION OF YIELD MANAGEMENT IN HOTELS: PRE-COVID-19 \\ STAGE AND EXPECTATIONS IN THE CURRENT PANDEMIC \\ CONTEXT \\ CAROLINA PASCIARONI' \\ SILVINA ELÍAS' \\ JOSÉ IGNACIO DIEZ'
}

IUNIVERSIDAD NACIONAL DEL SUR, BAHÍA BLANCA, ARGENTINA.

DATA DE SUBMISSÃO: 08/03/2021 - DATA DE ACEITE: 01/07/2021

RESUMEN: La incertidumbre sobre la recuperación de la demanda provocada por la pandemia COVID-19 en combinación con las nuevas erogaciones que deben afrontar los hoteles para cumplir con los protocolos sanitarios y adquirir soluciones tecnológicas que atenúen el riesgo de contagio, constituyen factores que revalorizan la adopción de la filosofía Yield Managment (YM). Partiendo de la necesidad de profundizar en el conocimiento empírico sobre la implementación del YM en países de menor desarrollo, el estudio tiene como objetivo: 1) analizar las características de los hoteles y su relación con el conocimiento y adopción del YM para la fijación de tarifas en Argentina e 2) identificar cambios en su adopción y/o uso en el contexto de pandemia. El estudio de caso constituye la metodología empleada y se centra en los hoteles de 3 y 4 estrellas de la ciudad de Bahía Blanca. La información se recopiló a través de entrevistas semi-estructuradas presenciales a los encargados de los seis hoteles de 4 y 3 estrellas (enero a marzo 2019), y encuestas difundidas vía e-mail a los mismos alojamientos (octubre a noviembre de 2020). De acuerdo al análisis de esta información, ciertos rasgos de los hoteles se relacionan con el conocimiento y efectiva adopción del YM. El estudio muestra que la crisis COVID-19 no promueve la adopción del YM.

PALABRAS CLAVE: hoteles; gestión del ingreso; Yield Management; Revenue Management; COVID-19; Argentina.

Carolina: Asistente de docencia, Departamento de Economía, Universidad Nacional del Sur (UNS), Bahía Blanca, Argentina. Licenciada y Doctora en Economía por la Universidad Nacional del Sur, Bahía Blanca, Argentina. E-mail: carolina.pasciaroni@uns.edu.ar. Orcid: https://orcid.org/0000-0003-2960-998X

Silvina: Profesora, Departamento de Economía, Universidad Nacional del Sur (UNS), Bahia Blanca, Argentina - Investigadora del Instituto de Investigaciones Económicas y Sociales del Sur (UNS-CONICET), Bahia Blanca, Argentina). Licenciada y Magister en Economía por la Universidad Nacional del Sur, Bahía Blanca, Argentina. E-mail: selias@uns.edu.ar. Orcid: https://orcid.org/0000-0001-7750-1821

José Ignacio: Profesor, Departamento de Economía, Universidad Nacional del Sur (UNS), Bahia Blanca, Argentina - Investigador del Instituto de Investigaciones Económicas y Sociales del Sur (UNS-CONICET), Bahia Blanca, Argentina). Licenciado y Doctor en Economía por la Universidad Nacional del Sur, Bahía Blanca, Argentina.E-mail: jdiez@uns.edu.ar. Orcid: https://orcid.org/0000-0001-6027-2014 
RESUMO: A incerteza sobre a recuperação da demanda provocada pela pandemia COVID-19 em combinação com os novos gastos que os hotéis devem enfrentar para cumprir os protocolos de saúde e adquirir soluções tecnológicas que mitiguem o risco de contágio, são fatores que reavaliam a adoção do Yield Management (YM) filosofia. Partindo da necessidade de aprofundar o conhecimento empírico sobre a implementação do YM em países menos desenvolvidos, o estudo visa: 1) analisar as características dos hotéis e sua relação com o conhecimento e adoção do YM para fixação de tarifas na Argentina e 2) identificar mudanças em sua adoção e / ou uso no contexto de uma pandemia. O estudo de caso constitui a metodologia utilizada e centra-se nos hotéis de 3 e 4 estrelas da cidade de Bahía Blanca. As informações foram coletadas através de entrevistas semiestruturadas face a face com os gestores dos seis hotéis de 4 e 3 estrelas (janeiro a março de 2019), e inquéritos divulgados via e-mail para os mesmos alojamentos (outubro a novembro de 2020). Conforme a informações analisadas, algumas características dos hotéis estão relacionadas ao conhecimento e adoção efetiva do YM. O estudo mostra que a crise de COVID-19 não promove a adoção do YM.

PALAVRAS-CHAVE: hotéis; gestão de receitas; Yield Management; Revenue Management; COVID-19; Argentina

\begin{abstract}
The uncertainty surrounding the recovery of demand, caused by the COVID-19 pandemic, combined with the new expenditures that hotels need to bear in order to comply with health protocols and acquire technological solutions to mitigate the risk of contagion, are factors that have led to a reevaluation of the adoption of the Yield Management (YM) philosophy. Based on the need to deepen empirical knowledge on the implementation of YM in less developed countries, this study aims: 1) to analyse the characteristics of hotels and how these relate to the knowledge and adoption of YM for setting rates in Argentina and 2) to identify changes in the adoption and/or use of YM in the context of the pandemic. The methodology used is a case study of 3- and 4-star hotels in the city of Bahía Blanca, Argentina. The information was collected through semi-structured face-to-face interviews with the managers of six hotels, conducted between January and March 2019, and surveys which were sent to the managers via email between October and November 2020. According to the analysis, there are certain characteristics of the hotels that are related to the knowledge and effective adoption of YM. The study shows that the COVID-19 crisis has not led to the increased adoption of YM.
\end{abstract}

KEYWORDS: hotels; Yield Management; Revenue Management; COVID-19; Argentina

\title{
INTRODUCCIÓN
}

La crisis acelerada y sin precedentes del turismo a nivel mundial provocada por la pandemia COVID-19 induce a repensar el modelo de negocios, las estrategias de innovación y la progresiva digitalización de las firmas pertenecientes al sector (UNWTO, 2020a; OECD, 2020; Shin y Kang, 2020; Kussmann, 2020). En este nuevo escenario, las innovaciones en el sector alojamiento se representan en la adopción de nuevas tecnológicas automatizadas y robóticas tendientes a reducir las interacciones personales y mejorar la higiene de las instalaciones (Kussmann, 2020; Shin y Kang, 2020; Jiang y Wen, 2020). Los sistemas check-in móviles, las llaves digitales, el reconocimiento facial y los sistemas de limpieza robotizados constituyen algunos ejemplos de innovaciones recientemente implementadas por las grandes cadenas hoteleras internacionales.

Por otra parte, la incertidumbre sobre la recuperación de la demanda en combinación con las erogaciones que deben afrontar los hoteles en relación a la adquisición de nuevas soluciones tecnológicas y/o el cumplimiento de los protocolos sanitarios, constituyen factores que revalorizan la adopción de prácticas back-office de gestión de ingresos. En esta dirección, 
el Yield Management (YM), también denominado Revenue Management (RM), contempla un conjunto adecuado de herramientas orientadas a maximizar los ingresos y los beneficios de las firmas prestadoras de servicios que operan con capacidad relativamente fija e inventario perecedero, tales como las empresas hoteleras y de transporte aéreo.

Siguiendo a Ivanov (2014, p. 8), Kimes (1989) y Kimes y Wirtz (2003), el YM se define como un sistema de herramientas y acciones dedicadas al logro del nivel óptimo de ingresos netos y ganancias operativas brutas de un hotel mediante la oferta del producto adecuado, a los clientes adecuados, a través del canal de distribución adecuado, en el momento adecuado, al precio adecuado y con la comunicación adecuada. Desde una perspectiva más amplia, el YM no solo es conceptualizado como un sistema de herramientas sino como una filosofía de gestión basada en el análisis de la información y orientada a maximizar el beneficio y el valor de la relación con el cliente mediante una gestión eficaz de precios e inventario (Abad et al., 2019; Talón Ballestero et al., 2008). En el contexto actual de incertidumbre sobre la recuperación de los niveles de ocupación, la posible guerra de precios entre competidores, el cambio en la segmentación, la pérdida de relevancia de los datos históricos ante el carácter extraordinario de la crisis, el YM permite flexibilizar y adaptar las tarifas a esta dinámica cambiante, monitorizar tanto las reservas recibidas como las cancelaciones y sustituir tarifas no reembolsables por tarifas semiflexibles (Chicheri, 2020).

Si bien diversos estudios abordan la implementación del YM en relación a las características de los hoteles (Murimi, et al., 2021; Abad et al., 2019; Xiaowen et al.; 2013; Talón Ballestero y González-Serrano, 2012 y 2013; Ivanov y Ayas; 2017), las investigaciones empíricas se centran mayoritariamente en países desarrollados. Tal como indican Ivanov y Ayas (2017, p. 137), mientras que la gestión de ingresos hoteleros ha crecido enormemente como campo de investigación durante los últimos 20 años y su implementación en economías desarrolladas es ampliamente reconocida e investigada, su adopción por parte del sector hotelero en Europa Central y Oriental, Medio Oriente, América y África no ha recibido mucha atención en la literatura académica especializada, con algunas excepciones. En el caso de América Latina, Boaria y Gadotti dos Anjos (2014) analizan los efectos del YM sobre el desempeño económico de un hotel localizado en Brasil.

De este modo, partiendo del rol asignado a la innovación, específicamente la adopción del YM como herramienta adecuada para responder a la crisis del COVID-19, sumado a la necesidad de profundizar en los determinantes de la implementación del YM en hoteles localizados en países de menor grado de desarrollo, el presente estudio tiene como objetivo: 1) analizar las características de los alojamientos y su relación con el conocimiento y adopción del YM para la fijación de tarifas entre los hoteles de 3 y 4 estrellas de la ciudad de Bahía Blanca (Argentina) e 2) identificar cambios en su adopción y/o uso en virtud del nuevo escenario que plantea la pandemia COVID-19. El análisis propuesto, de carácter descriptivo y exploratorio, se centra en el YM orientado a la fijación de tarifas y procurará verificar si algunos de los condicionantes señalados en la literatura sobre adopción del YM en países desarrollados también se evidencian en el centro urbano mencionado.

Teniendo en cuenta que la metodología del estudio de caso es empleada por distintos estudios sobre implementación del YM (Chávez Miranda y Ruiz Jiménez, 2005; Chávez Miranda 
et al., 2014; Okumus, 2004), la selección de Bahía Blanca (Argentina) como caso de estudio responde a distintos factores. Por un lado, esta ciudad de tamaño poblacional medio constituye un centro político-administrativo, comercial y de servicios de salud y educación universitaria para una amplia zona de influencia, a la vez que, se desempeña como nodo de comunicación y transporte y centro de escala y distribución de turistas y visitantes hacia los centros turísticos de playa y montaña cercanos (Schroeder y Formiga, 2009; Pinassi y Ercolani, 2012). Por otro lado, el estudio de la oferta hotelera de la ciudad permite indagar en la adopción del YM por parte de alojamientos que responden a la categoría de pequeñas y medianas empresas (PYMES), no pertenecen a una gran cadena internacional y no se encuentran localizados en grandes urbes. En los alojamientos que cumplen con estas características, la adopción del YM resulta un poco más tardía. Al respecto, Talón Ballestero et al. (2008) señalan que, en España, el YM fue adoptado primeramente por cadenas hoteleras internacionales y en las grandes urbes como Madrid y Barcelona y luego, progresivamente, por parte de los hoteles de menor tamaño e independientes.

El artículo se estructura de la siguiente manera. En la primera sección, se revisa la literatura referida a innovación en hotelería y los aportes relativos a la implementación del YM. En la próxima sección se exponen consideraciones metodológicas, se describe el área de estudio y los hoteles de tres y cuatro estrellas bajo análisis y se indica la información relevada mediante entrevistas presenciales (período previo al COVID-19) y encuestas on-line (período actual de crisis COVID-19). En la tercera sección, se muestran los resultados encontrados para los dos períodos definidos en el análisis. En la cuarta y última sección, se presenta una síntesis de los resultados, limitaciones del estudio y futuros lineamientos.

\section{FUNDAMENTACIÓN TEÓRICA}

Siguiendo a Jones (2000); Talón Ballestero et al. (2008) y Chávez Miranda y Ruiz Jiménez (2005), existen varias definiciones de Yield Management (YM). La definición más difundida considera al YM como el proceso de asignación de una unidad de capacidad al tipo de cliente adecuado, en el momento adecuado y al precio adecuado con el objetivo de maximizar el ingreso (Kimes, 1989: 15; Smith et al., 1992). De acuerdo a Jones (2000, p. 88), el YM se define como un sistema para maximizar la rentabilidad de los hoteles, identificando la rentabilidad de los segmentos de demanda, estableciendo precios, creando reglas de descuento para su aplicación en los avanzados procesos de reserva y monitoreando la efectividad de esas reglas.

La aplicación del YM resulta apropiada en sectores donde las firmas operan con capacidad relativamente fija; cuentan con la posibilidad de segmentar el mercado; poseen inventario perecedero; posibilidad de vender el producto anticipadamente; demanda variable en función del momento en el tiempo, aunque predecible; costes marginales de venta bajos y costes marginales de producción altos (Kimes 1989; Kimes y Chase, 1998). El sector hotelero, al igual que la industria área, responde a estos requisitos, a la vez que, presenta características diferenciales, tales como las estancias de múltiples noches; el efecto multiplicador entre los servicios de alojamiento y servicios de restauración; el mayor tiempo de antelación de realización de las reservas y los diferentes horarios de arribo por parte de los clientes (Jones, 2000, ver revisión Chávez Miranda y Ruiz Jiménez, 2005). 
Inicialmente, el YM fue implementado por la industria área en la década de 1970 frente a la creciente competencia derivada de la desregulación del sector, y adoptado por la industria hotelera americana en los años 80s (Kimes, 1989; Talón Ballestero et al., 2008). Más allá del entorno cada vez más competitivo y los beneficios asociados a su implementación, los avances tecnológicos constituyeron una motivación adicional para su creciente adopción (Talón Ballestero et al., 2008). Es preciso indicar que, la innovación en el sector alojamiento se encuentra ampliamente asociada a la adopción de avances tecnológicos en el campo de las TICs (Orfila-Sintes y Mattsson, 2009; Nieves et al., 2014; Nieves y Osorio; 2019; Benckendorff et al., 2019; Fernández et al., 2011; Sigala, 2003). Si bien, las TICs no se difundieron rápidamente en el sector hotelero, actualmente es considerado un sector intensivo en información (Law y Jogaratnam, 2005; Benckendorff et al., 2019).

El uso de información y softwares específicos de gestión de ingresos son algunos de los elementos que componen el YM. De acuerdo a Ivanov (2014), el YM puede ser definido como un sistema integrado por cuatro elementos estructurales: datos e información; centros o fuentes de ingresos del hotel; software de YM y herramientas de YM. En relación a este último elemento, la gestión de ingresos implica la utilización de diferentes herramientas divididas en 1) instrumentos de precio o pricing, 2) instrumentos de cantidad o no-princing, tales como control de inventarios, overbookings, controles sobre la duración de las estancias e 3) instrumentos combinados, representados en la gestión de canales de distribución y la asignación óptima de habitaciones. Las herramientas de fijación de precios, de interés para el presente estudio, incluyen la discriminación de precios, el uso de tarifas restringida, la fijación de tarifas dinámicas, las garantías de precio más bajo y otras técnicas que influyen directamente en los precios de un hotel (su nivel, estructura, presentación y reglas de precios).

La aplicación del YM requiere de una gran cantidad de datos relativos a diferentes métricas de ingresos (ADR, RevPAR, GOPPAR), futuras reservas, venta de servicios adicionales, tarifas y estrategias de la competencia, información sobre cambios en la legislación, eventos especiales en el lugar de destino (Ivanov, 2014, p. 26). El uso de softwares específicos de YM facilita la recopilación, el almacenamiento y el análisis de datos, y, en consecuencia, incrementa la eficiencia en la toma de decisiones en relación a las tarifas, las cuales se vuelven flexibles según la fecha, el tipo de cliente, el canal de distribución y el tipo de servicio (Ivanov 2014; Cheraghi et al., 2010; O' Connor y Murphy, 2008; Johnson, 2006).

De todos modos, los beneficios derivados del YM no sólo dependen del uso de sistemas de información y tecnología sino también a la experiencia y conocimiento empresarial (Ivanov, 2014; O' Connor y Murphy, 2008). En un contexto de creciente complejidad en la toma decisiones, se acentúa la necesidad de recursos humanos con habilidades, especialmente analíticas y comunicacionales, y puestos de trabajo en el interior de los hoteles específicamente dedicados a la gestión de ingresos (Kimes, 2017; Ferguson y Smith, 2014; Erdem y Jiang, 2016). Debido a restricciones presupuestarias, los hoteles de tamaño más pequeño, no pueden asignar un gerente de tiempo completo para esta función (Ferguson y Smith, 2014).

En cuanto a los factores que inciden en la adopción del YM, la literatura sobre innovación en hoteles ofrece un primer esquema para su análisis. Siguiendo Orfila-Sintes (2003 y 2009) y Martínez-Ros y Orfila-Sintes (2009), los determinantes de la innovación en hotelería 
pueden clasificarse en: 1) factores asociados a las características del hotel como su capacidad física o tamaño, la estructura de la propiedad y el grado de dependencia de otras estructuras organizativas, la modalidad de gestión del hotel y los servicios adicionales que presta; 2) factores asociados a las características de la demanda y la comercialización de los servicios, tales como el motivo del viaje de los huéspedes, la estacionalidad y el grado de uso de las instalaciones, los canales utilizados para reservar habitaciones y 3) la estrategia de mercado en relación a la diferenciación de los servicios respecto a los competidores. A estos determinantes se suma el conocimiento y las habilidades de los empleados, considerado fuente clave de innovación para los hoteles (Nieves et al., 2014; Nieves y Segarra-Ciprés, 2015; Dhar, 2015; Ottenbacher y Harrington, 2010).

El acceso a financiamiento y aspectos organizativos vinculados a la predisposición al cambio y rigideces, son factores alternativos que condicionan la decisión de innovar de las empresas hoteleras (López-Fernández et al., 2009). Orfila-Sintes y Mattsson (2009) encuentran que el tamaño, la prestación de servicios adicionales, el motivo de viaje, y la capacitación de los recursos humanos tienen un efecto positivo sobre la probabilidad de obtener innovaciones back-office en los hoteles. Es preciso destacar que las empresas no innovan solas; la innovación es un proceso que depende de la adquisición de conocimiento e información proveniente de distintos agentes (Lundvall, 2010). En el caso del sector hotelero, Nieves et al. (2014) y Nieves y Diaz-Meneses (2018), Nieves y Segarra-Ciprés (2015), ofrecen evidencia empírica a favor del impacto positivo que los vínculos con agentes externos tienen sobre la innovación en hoteles.

Por su parte, Fernández et al. (2011) ofrecen un marco conceptual para analizar los condicionantes externos que influyen en la innovación hotelera y la inversión en TICs, diferenciando los mismos en: 1) políticas de promoción de la innovación en turismo y hotelería; 2) factores económicos tales como crisis y ciclos económicos; 3) factores sociodemográficos representados en cambios de hábitos de consumo y nuevos segmentos de mercado; 4) nuevas tecnologías traducidas en innovaciones de producto, proceso, organizacionales o de marketing en el sector turístico; 5) factores medioambientales ejemplificados en legislación para regular la contaminación y preservar el medioambiente; 6) cambios en la infraestructura turística y nuevas infraestructuras de comunicación. La crisis de COVID-19 puede ser considerada un factor externo excepcional que incide en un gran número de aspecto de la industria hotelera, entre los que se encuentran la diversificación de los servicios ofrecidos y los canales de distribución adoptados por los hoteles; la oferta de servicios de alojamiento que aseguren higiene y distanciamiento social, la transformación digital e inteligente y la reorganización del mercado (Hao et al., 2020; Shin y Kang, 2020; Jiang y Wen, 2020). Un aspecto que revaloriza la utilidad del YM en este contexto de crisis deriva de la búsqueda por parte de los hoteleros de la maximización de los ingresos por metro cuadrado por minuto (Gallen, 2020 en Hao et al., 2020).

En relación al YM específicamente, diversos estudios dan cuenta de la importancia de la categoría, el tamaño y/o la pertenencia a una cadena como determinantes de la adopción del YM (Abad et al., 2019; Xiaowen et al.; 2013; Talón Ballestero y González -Serrano, 2012 y 2013; Ivanov y Ayas; 2017). La existencia de personal calificado a tiempo completo, representado en la figura del Yield Manager, también es un determinante de la implementación exitosa del YM (Abad et al., 2019; Zeni, 2003; Kimes, 2017). Chávez Miranda et al. (2014) en su análisis referido a 
hoteles de 4 y 5 estrellas de Sevilla, encuentran que la formación del personal y la no disposición de información interna constituyen los obstáculos iniciales. Tras la aplicación del YM, los obstáculos se concentran en la falta de formación oportuna por parte del personal del hotel y la falta de un software de YM adaptado totalmente al sector. Okumus (2004) señala la falta de conocimiento a la escasa formación y cultura sobre la gestión de ingresos, la baja adaptación de un software para su aplicación, su costo de instalación y la resistencia por parte de directivos a invertir en el proyecto de software.

Figura 1: Condicionantes internos y externos respecto a la adopción de YM en hotelería

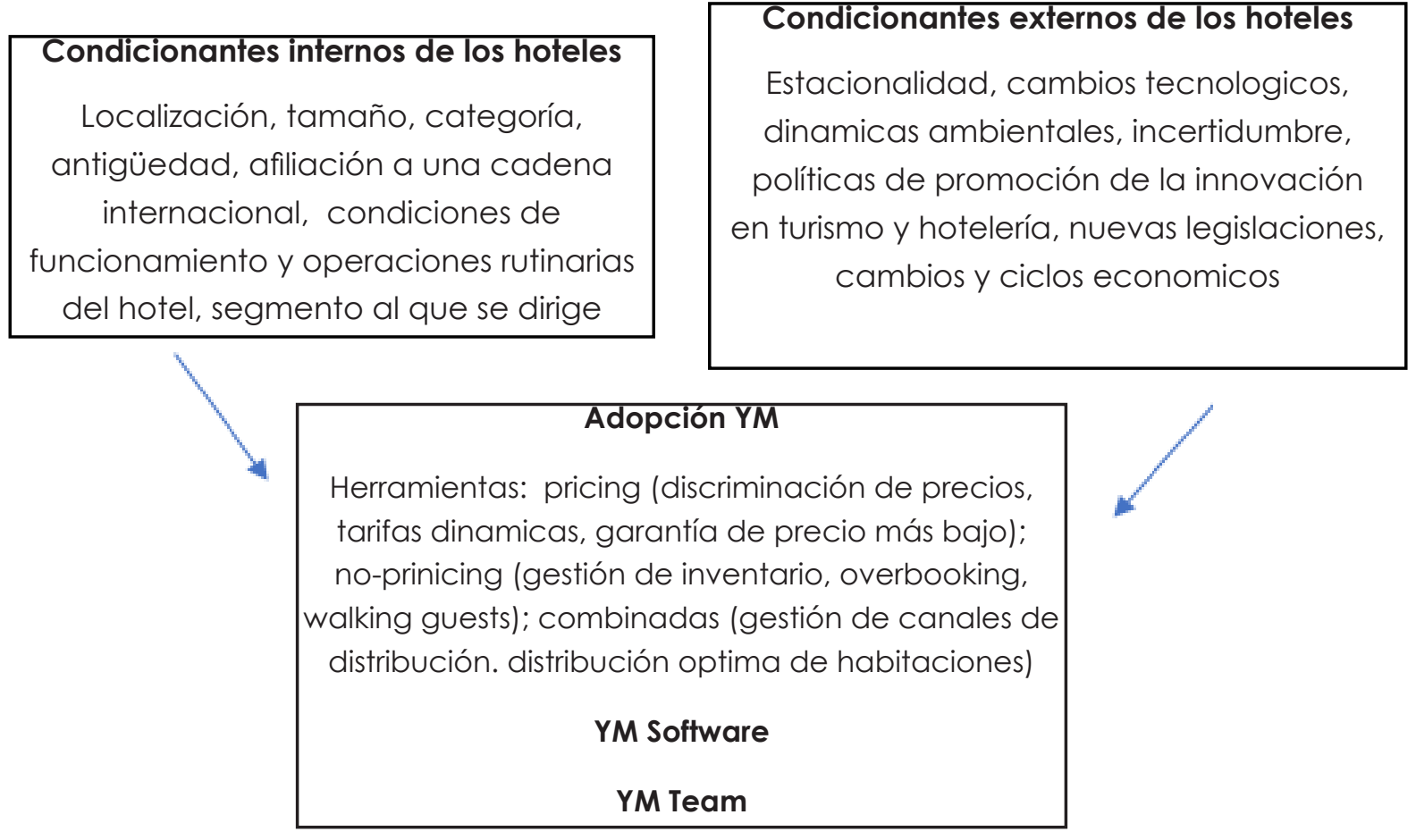

Fuente: elaboración propia en base Murimi, et al., 2021; Fernández et al. (2011); Ivanov (2014); Talón-Balleteros, et al. (2012).

\section{METODOLOGÍA}

Caso de estudio. Al igual que en estudios previos sobre YM (Chávez Miranda y Ruiz Jiménez, 2005; Chávez Miranda et al., 2014; Okumus, 2004), se emplea el estudio de caso como técnica metodológica. De acuerdo a Yin (2011) se trata de un método empírico usado en las ciencias sociales para contribuir al conocimiento sobre un fenómeno contemporáneo dentro de su contexto de la vida real. Este método no supone el uso de un solo tipo de evidencia, por el contrario, se basa tanto en información de tipo cuantitativa como cualitativa, y su alcance puede ser exploratorio, descriptivo y explicativo.

La ciudad de Bahía Blanca (Argentina) y su oferta hotelera de tres y cuatro estrellas, constituye el caso bajo estudio. Esta localidad se encuentra ubicada a los $38^{\circ} 44^{\prime}$ de latitud sur y $62^{\circ} 16^{\prime}$ longitud oeste, en el sudoeste de la Provincia de Buenos Aires. Según el último Censo Nacional de Población y Vivienda (año 2010), en la ciudad residen 301.531 habitantes, situándose 
en la categoría de centro urbano de tamaño medio según la estructura urbana del país. Su localización geográfica, estructura productiva e infraestructura y transporte y comunicaciones convierten a la ciudad en un centro de gravitación regional (Gorenstein et al., 2012). En esta dirección, Bahía Blanca se ha consolidado como centro comercial, financiero y de prestación de servicios de salud y educativos para una gran zona de influencia. Su sector industrial se integra por un conjunto de empresas asociadas a la actividad petroquímica y agroindustrial. La ciudad cuenta con un puerto local, único de aguas profundas del país, que permite la salida de granos, oleaginosas hacia mercados internacionales. Además, se desempeña como nodo de comunicaciones, conectada a las principales ciudades del país a través de un aeropuerto y una red de rutas nacionales y provinciales.

Desde la perspectiva del turismo, Bahía Blanca integra el conjunto de localidades que reúnen el $80 \%$ de las plazas disponibles de la Provincia de Buenos Aires, junto a Mar del Plata, Pinamar, Tandil y Villa Gesell (INDEC, 2019). Es preciso indicar que, la estadía promedio en la ciudad es de 1,4 noches, resultando inferior a la estadía registrada por las restantes ciudades mencionadas (INDEC, 2019), las cuales reciben turistas por viajes de ocio en función de sus atractivos naturales de playa y montaña. Actualmente, la oferta hotelera de 3 y 4 estrellas de la ciudad se compone de siete hoteles: tres hoteles pertenecen a la categoría 3 estrellas, tres hoteles a la categoría 3 estrellas superior, y un hotel a la categoría 4 estrellas superior. Solo tres de este conjunto hoteles pertenecen a una cadena. Se trata de las cadenas Hoteles Land y Hoteles Austral, ambas de origen y alcance nacional.

Recopilación de información. El proceso de recopilación fue llevado a cabo en das etapas con distintos instrumentos de obtención de la información: entrevistas presenciales durante etapa anterior a COVID-19 y encuestas enviadas vía e-mail en etapa de pandemia. En la primera etapa, durante los meses de enero y febrero del año 2019, se efectuaron entrevistas semi-estructuradas presenciales a los seis hoteles que componían la oferta hotelera de 3 y 4 estrellas de la ciudad a principios de ese año. Las entrevistas fueron realizadas al personal a cargo de los hoteles, previo contacto telefónico con los mismos. En base a la distinción entre conocimiento y uso efectivo del YM (Chávez Miranda y Ruiz Jiménez, 2005), las entrevistas semiestructuradas se concentraron en recopilar información sobre: 1) las fuentes de información empleadas por los hoteles para actualizarse sobre nuevos métodos, prácticas y sistemas para la fijación de tarifas y, su conocimiento del termino y la definición del YM y 2) la aplicación efectiva del YM para la fijación de tarifas. En la segunda etapa, correspondiente al período de pandemia en octubre de 2020, se enviaron encuestas vía mail al mismo conjunto de hoteles de 3 y 4 estrellas. Los formularios en formato Word enviados se diseñaron y estructuraron en torno a la obtención de información sobre cambios realizados y proyectados en contexto de crisis COVID-19, entre los que se encuentra la implementación del YM en los hoteles que no adoptaban el YM antes de la crisis COVID-19 y la intensificación en el uso del YM como referencia exclusiva para la fijación de tarifas por parte de los hoteles que ya adoptaban este sistema de gestión de ingresos. Sólo un hotel no respondió la encuesta enviada por e-mail; el resto de los hoteles completó y envío el formulario durante los meses de octubre y noviembre de 2020, previo llamado telefónico en algunos casos. Por último, la información recopilada para los dos períodos y los distintos hoteles, fue ordenada y agrupada por categoría de análisis 
utilizando un procesador de textos para facilitar el posterior análisis comparativo entre hoteles y etapas y la formulación de relaciones.

Tabla 1: Información relevada en entrevistas semi-estructuradas y encuestas

\begin{tabular}{|c|c|}
\hline \multicolumn{2}{|c|}{\begin{tabular}{c|} 
Caracterización de los hoteles \\
\hline Categoría - Localización en la ciudad- Año de Inicio - Tipo de Empresa- Número de empleados- \\
Número de habitaciones y plazas
\end{tabular}} \\
\hline \multicolumn{2}{|c|}{ Aplicación de prácticas de segmentación y overbooking } \\
Reservas y canales de distribución
\end{tabular}

Fuente: Elaboración Propia. (*) Otras instituciones: Federación Empresaria Hotelera Gastronómica de la Provincia de Buenos Aires (FEHGPBA) y a la Federación Empresaria Hotelera Gastronómica de la República Argentina (FEHGRA).

\section{RESULTADOS}

\section{Período 1: PRe-PANDEMia COVID-19}

La información primaria recopilada con anterioridad a la crisis COVID-19, permitió agrupar a los hoteles de 3 y 4 estrellas de la ciudad según su conocimiento y aplicación efectiva del YM para la fijación de tarifas (Tabla 2). De este modo, se identificó un primer grupo, conformado por tres hoteles de categoría 3 y 4 estrellas superior y pertenecientes a una cadena, los cuales declararon tener conocimiento sobre el término y definición de YM e implementar esta filosofía de gestión. En el extremo opuesto, se delimitó un segundo grupo 
conformado por tres hoteles de categoría 3 estrellas y 3 estrellas superior, y no afiliados a una cadena. Estos hoteles declararon no conocer el sistema de gestión de ingresos bajo estudio, y por lo tanto tampoco aplicarlo. Asimismo, se identificó un hotel pequeño, de 3 estrellas y no perteneciente a una cadena, cuyo entrevistado expresó que el hotel tiene conocimiento sobre el YM, aunque no la aplica.

Tabla 2: Período 1: pre-pandemia COVID-19

\begin{tabular}{|c|c|c|c|}
\hline \multirow{2}{*}{ Hoteles } & Grupo 1 & Grupo 2 & Grupo 3 \\
\hline & Conoce y aplica el YM & No conoce el YM & Conoce y no aplica el YM \\
\hline Categoría & $\begin{array}{c}4 \text { estrellas superior ( } 1 \text { hotel) } \\
3 \text { estrellas superior } \\
\text { ( } 2 \text { hoteles) }\end{array}$ & $\begin{array}{c}3 \text { estrellas superior } \\
\text { ( } 1 \text { hotel) } \\
3 \text { estrellas ( } 1 \text { hotel) }\end{array}$ & 3 estrellas ( 1 hotel) \\
\hline Cadena & Pertenecen a una cadena & $\begin{array}{l}\text { No pertenecen a una } \\
\text { cadena }\end{array}$ & $\begin{array}{l}\text { No pertenece a una } \\
\text { cadena }\end{array}$ \\
\hline Tamaño & $\begin{array}{c}2 \text { hoteles de mayor tamaño } \\
\text { de la ciudad } \\
\text { (108 habitaciones) } \\
1 \text { hotel tamaño medio } \\
\text { (42 habitaciones) }\end{array}$ & $\begin{array}{l}\text { Hoteles de tamaño } \\
\text { grande (100 y } 80 \\
\text { habitaciones) }\end{array}$ & $\begin{array}{l}\text { Hotel pequeño } \\
\text { (16 habitaciones) }\end{array}$ \\
\hline Antigüedad & Década 2000, 1990 y 1950 & Década 1990 y 1920 & Década de 2000 \\
\hline $\begin{array}{l}\text { Demanda-Tipo } \\
\text { de huéspedes }\end{array}$ & $\begin{array}{c}\text { Huéspedes frecuentes por } \\
\text { motivo laboral } \\
\text { Huéspedes de origen } \\
\text { regional, nacional y } \\
\text { extranjeros }\end{array}$ & $\begin{array}{l}\text { Huéspedes frecuentes por } \\
\text { motivo laboral } \\
\text { Huéspedes de origen } \\
\text { regional, nacional }\end{array}$ & $\begin{array}{l}\text { Huéspedes frecuentes por } \\
\text { motivo laboral } \\
\text { Huéspedes de origen } \\
\text { regional, nacional }\end{array}$ \\
\hline Segmentación & \multicolumn{3}{|c|}{ Segmentan mercado } \\
\hline Overbooking & Sobrevenden & $\begin{array}{c}\text { Solo hotel } 3 \text { estrellas } \\
\text { superior }\end{array}$ & No sobrevende \\
\hline Reservas & \multicolumn{3}{|c|}{ Utilizan sistema reserva } \\
\hline \multicolumn{4}{|c|}{ Fuentes de información } \\
\hline Disponibles (*) & \multicolumn{3}{|c|}{ Uso por parte de todos los hoteles } \\
\hline $\begin{array}{c}\text { Cámaras } \\
\text { empresariales }\end{array}$ & $\begin{array}{l}\text { Uso por parte de } \\
\text { todos los hoteles }\end{array}$ & $\begin{array}{c}\text { Solo uso por parte hotel } 3 \\
\text { estrellas }\end{array}$ & $\begin{array}{l}\text { Usa este tipo de } \\
\text { fuente }\end{array}$ \\
\hline Otros hoteles & $\begin{array}{l}\text { Uso por parte de } \\
\text { todos los hoteles }\end{array}$ & $\begin{array}{c}\text { Solo uso por parte } \\
\text { hotel } 3 \text { estrellas }\end{array}$ & $\begin{array}{l}\text { No usa este tipo de } \\
\text { fuente }\end{array}$ \\
\hline Otras fuentes & $\begin{array}{l}\text { Uso por parte de } \\
\text { todos los hoteles }\end{array}$ & $\begin{array}{c}\text { Solo uso por parte hotel } 3 \\
\text { estrellas }\end{array}$ & $\begin{array}{l}\text { Usa este tipo de } \\
\text { fuente }\end{array}$ \\
\hline
\end{tabular}

Fuente: Elaboración propia (*) Fuentes Disponibles: información disponible en Internet, publicaciones, ferias.

Respecto a la implementación efectiva del YM para la fijación de tarifas, a partir de la Tabla 2 se pueden derivar consideraciones relativas a las características de los hoteles:

1) Categoría. Los hoteles que declararon conocer y adoptar el YM, alcanzan las categorías más altas dentro de la oferta hotelera de la ciudad. Entre los cuatro hoteles que indicaron no implementar el YM, sólo uno corresponde a la categoría 3 estrellas superior y los restantes alojamientos pertenecen a la categoría 3 estrellas. 
2) Afiliación a una cadena. A diferencia de los hoteles que declararon implementar el YM, los cuales forman parte de una cadena de origen y alcance nacional, la no pertenencia a una cadena es la característica común entre los alojamientos que no emplean el YM.

3) Tamaño. El número de plazas y habitaciones es un aspecto que no permite diferenciar entre hoteles que aplican el YM (Grupo 1) y hoteles que no aplican la gestión de ingresos (Grupo 2 y 3). Si bien, el primer grupo incluye a los hoteles de mayor tamaño de la ciudad (108 habitaciones), también forma parte de este grupo, un hotel de tamaño medio (42 habitaciones). Además, dos de los hoteles que indicaron que no adoptan el YM cuentan con 100 y 80 habitaciones, respectivamente, resultando alojamientos de dimensiones considerables en la oferta hotelera de 3 y 4 estrellas de la ciudad.

4) Antigüedad. Al igual que el tamaño, la antigüedad del hotel (año de inicio de sus actividades) es un aspecto que no permite diferenciar a los hoteles de acuerdo a su conocimiento y uso del YM. Los hoteles de mayor y menor antigüedad se encuentran tanto en el grupo de alojamientos que declararon hacer uso del YM como en el grupo que indicaron no adoptar el YM.

5) Demanda o tipo de huéspedes. Los huéspedes de todos los hoteles analizados se hospedan de forma frecuente principalmente por trabajo, salud, comercio y trámites administrativos. Se trata de clientes que residen en el país, provenientes de la Ciudad Autónoma de Buenos Aires, el sur del país y la zona de influencia de Bahía Blanca (Sudeste de la Provincia de Buenos Aires). Se señala que, entre los hoteles que declararon implementar el YM para la fijación de tarifas, los huéspedes también proceden del exterior del país.

6) Uso de fuentes de información para actualizarse respecto a prácticas y procesos de fijación de tarifas. Se destaca el uso de una mayor variedad de fuentes de información por parte de los hoteles que indicaron implementar el YM para la fijación de tarifas. Estos hoteles señalaron que utilizan información proveniente de cámaras empresariales; otros hoteles de la propia cadena y/o competidores; otras fuentes de información como huéspedes y proveedores e información disponible en Internet, revistas especializadas, ferias. Los hoteles que señalaron que no implementan el YM se caracterizan por utilizar un menor número de fuentes de información respecto a los hoteles que sí tienen conocimiento sobre el YM.

7) Reservas, segmentación y overbooking. Todos los hoteles entrevistados, indicaron que segmentan el mercado y recurren a canales de distribución disponibles en Internet tales como las plataformas Booking y Trivago. En general, los hoteles analizados consideran a los huéspedes frecuentes y a los grupos para diferenciar tarifas. Excepto dos, el resto de los hoteles mencionó llevar a cabo la práctica de overbooking. Uno de los hoteles señaló que esta práctica contra su política de fidelización de clientes. Es preciso indicar que, el overbooking constituye una práctica ampliamente aplicada en la industria hotelera y se define como la reserva de un número de habitaciones superior a la capacidad disponible del hotel (Ivanov, 2006).

Entre hoteles entrevistados que declararon conocer y aplicar el YM se observan los siguientes aspectos: 
- la antigüedad en la implementación del YM, la cual varía entre diez años y un año a la fecha de la realización de la entrevista.

- uso de softwares específicos: los tres hoteles que declararon aplicar el YM también indicaron que emplean softwares particulares a la actividad hotelera (ARION PMS y CLOCL PMS). Se trata de plataformas digitales de nueva generación para la gestión hotelera y cuentan con una gran cantidad de herramientas, entre ellas la inteligencia en la fijación de tarifas para mejorar la rentabilidad y productividad.

- personal idóneo y puesto del Yield Manager: ninguno de los hoteles que indicaron hacer uso del YM cuenta con la figura del Yield Manager. Por el contrario, el Jefe de ventas, el Jefe de Recepción, el Gerente o los propios encargados del hotel destinan parte de su tiempo a la gestión de ingresos.

- obstáculos y beneficios percibidos en su implementación: los tres hoteles que indicaron adoptar el YM no detectaron dificultades en su implementación y/o uso del YM, aunque señalaron que la cultura local en términos de la gran sensibilidad del huésped al cambio en las tarifas constituye una restricción.

Si bien la mayoría de los hoteles que declararon no aplicar el YM manifestaron no tener conocimiento previo del mismo, cuando se indicó al entrevistado sobre el concepto y características del YM, estos consideraron que podría ser una opción a implementar y que tal filosofía de gestión orientada a la fijación de tarifas maximizaría sus ingresos. Sin embargo, también señalaron que la situación económica del país (recesión, inflación, incertidumbre), los costos elevados del propio sector y la falta de personal idóneo constituyen restricciones para su pronta implementación.

\section{Período 2: contexto actual de Pandemia COVID-19}

A mediados de marzo de 2020, tras la caracterización del COVID-19 como pandemia por parte de la Organización Mundial de la Salud y de forma un poco más tardía respecto a países de Europa, América del Norte y Asia, en Argentina se dispusieron un conjunto de medidas restrictivas a la circulación interna de personas, el ingreso de pasajeros desde el exterior y el funcionamiento de actividades no esenciales. La Figura 2 señala las principales medidas adoptadas, las cuales dan cuenta de una extensión de casi 9 meses durante el año 2020 de la vigencia de limitaciones al normal funcionamiento del sector turístico tanto interno como internacional. 
Figura 2: Medidas adoptadas en Argentina durante 2020 frente a pandemia COVID-19

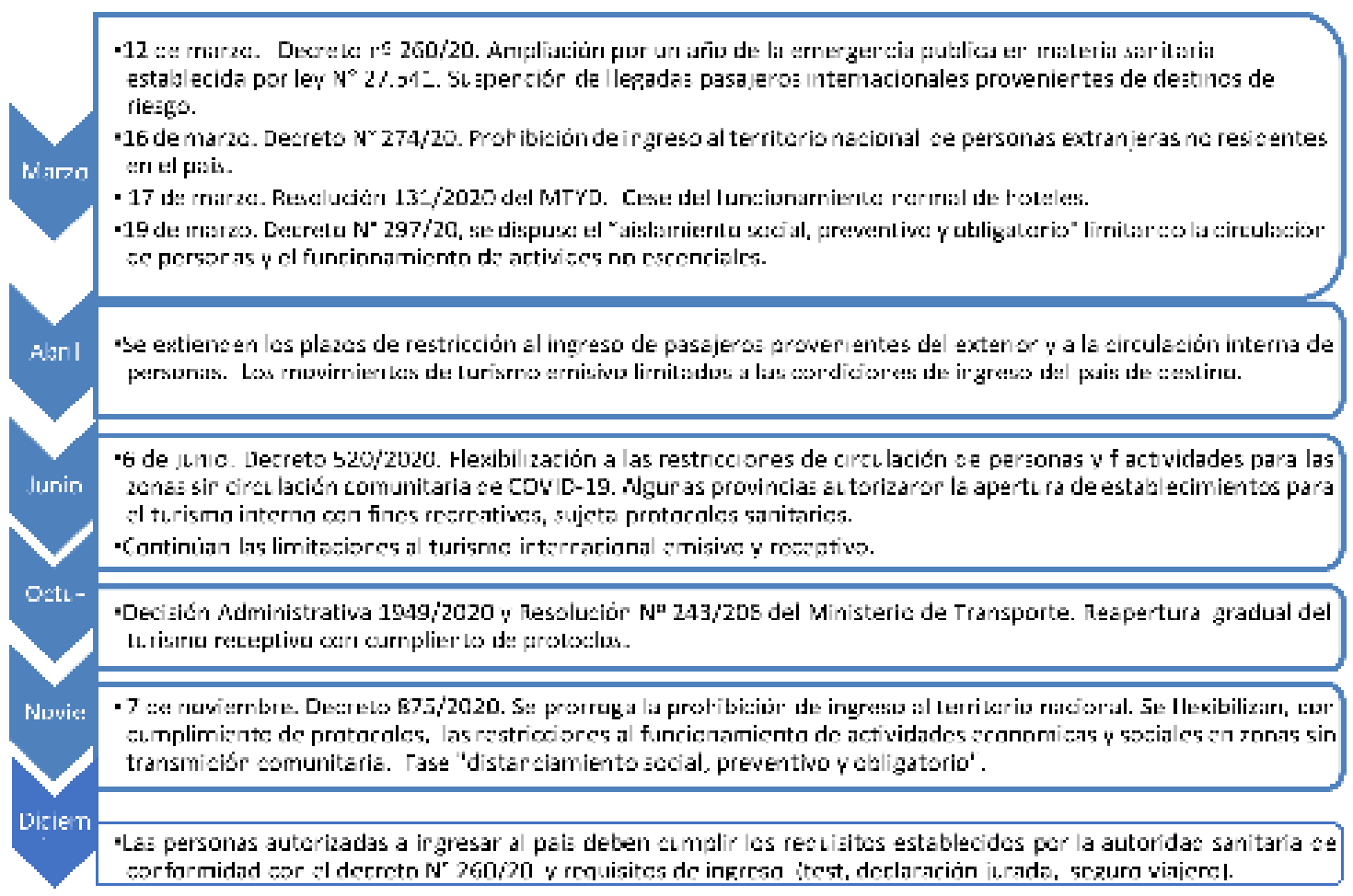

Fuente: Elaboración propia en base a INDEC (2020a, b y c) y Boletín Oficial de la República Argentina. Nota: se omiten las resoluciones y decretos que refrendan las medidas señaladas. MTyD: Ministerio de Turismo y Deportes de la Nación.

Respecto al sector hotelero, el Ministerio de Turismo y Deportes (MTyD) resolvió limitar en marzo de 2020 la actividad de los establecimientos hoteleros (Resolución N 131/202). En un primer momento, pudieron brindar alojamiento únicamente a los ciudadanos extranjeros varados en el país y a quienes se encontraban en situación de aislamiento obligatorio. A partir de junio del mismo año, en algunas provincias y localidades sin circulación comunitaria del virus, el Estado Nacional autorizó la apertura de establecimientos para el turismo interno con fines recreativos, limitándose esta actividad a turistas residentes de la localidad, corredor o provincia. De esta manera, las restricciones impuestas a inicios de 2020 fueron gradualmente flexibilizándose bajo cumplimiento de los protocolos sanitarios de los rubros vinculados al sector turístico.

El "Protocolo COVID-19 para Alojamientos Turísticos" elaborado por el MTyD en junio de 2020 y vigente a la actualidad, establece una serie de normas y recomendaciones relativas al distanciamiento social físico; la desinfección de superficies y ventilación de ambientes; la capacitación del personal; la organización del trabajo y aspectos relacionados con la atención al cliente tales como el uso de medios de pago online en el proceso de reserva, el adelanto por mail u otro medio electrónico la ficha de registro, junto con un cuestionario sobre COVID-19, el cumplimiento durante los procesos de check- in y check-out las pautas de higiene, y seguridad, la habilitación del pre-check-in virtual, una mayor frecuencia en la limpieza y desinfección, disposición del mobiliario de forma que contribuya al distanciamiento físico y la señalización de la circulación de las personas. 
El conjunto de restricciones indicadas en la Figura 2 tuvieron un alto impacto en el turismo a nivel nacional. De acuerdo a la Encuesta de Turismo Internacional (ETI), en el período enero-septiembre 2020, se observó un descenso interanual de 70,5\% en llegada de turistas no residentes y de 74,1\% en salidas de turistas hacia el exterior (INDEC 2020a). En base a la Encuesta de Ocupación Hotelera (EOH) de agosto de 2020, se evidenció una variación negativa de 98,1\% en el total de viajeros hospedados respecto al mismo mes del año anterior; un descenso interanual de $97,7 \%$ en el número de viajeros residentes, de $99,8 \%$ en el número de viajeros residentes, y una disminución interanual de $84,0 \%$ en el número de habitaciones ocupadas (INDEC 2020b). En la ciudad bajo estudio, Bahía Blanca, la tasa de ocupación de las habitaciones hotelera fue de tan solo 4,9\% en agosto de 2020, hospedando a 663 viajeros. Estas cifras contrarrestan con la tasa de ocupación registrada en agosto de 2019 de 42,8\% y los 9.374 viajeros hospedados en el mismo período (INDEC, 2020b).

En base a la información recopilada y sistematizada para Bahía Blanca, los hoteles de 3 y 4 estrellas encuestados manifestaron implementar reformas menores en espacios comunes para contribuir al distanciamiento físico entre personas, junto al planteamiento de nuevas estrategias de publicidad a través del uso de redes sociales; digitalización de reservas, checkin, check-out e internalización del servicio de limpieza. En relación a posibles cambios en la adopción del YM frente a la pandemia COVID-19, los alojamientos analizados que anteriormente no aplicaban el YM, continúan en la etapa COVID-19 sin emplear esta filosofía de gestión y no tienen planificada tampoco su adopción en el corto plazo. Los hoteles que previamente a la crisis COVID-19 adoptaban el YM, manifestaron que aporta herramientas óptimas para la toma de decisiones en el contexto de incertidumbre actual y demanda deprimida, la cual debe combinarse con acuerdos de precios entre los empresarios hoteleros.

Más allá de las innovaciones planeadas o en curso por parte de los hoteles para asegurar el distanciamiento social y el cumplimiento de los protocolos sanitarios, los hoteleros declararon no percibir la crisis de COVID-19 como una oportunidad para la adopción de innovaciones en general, y en particular, la implementación del YM. Las respuestas dadas por los encuestados refieren más a un comportamiento de tipo defensivo o de supervivencia empresarial que a la realización de inversiones asociadas a innovaciones. A modo de ejemplo, uno de los encuestados manifestó que "toda actividad de renovación y avance tecnológico se va a ver relegada por bastante tiempo. Las inversiones van a ser mínimas, solo para poder operar y brindar la calidad adecuada a cada hotel". En igual sentido, otra respuesta recibida indica que "el hotel que permanece abierto no hace más que endeudarse de una manera abismal que afectará, sin dudas, su desempeño a futuro, comprometiendo seriamente la capacidad de responder a cambios innovadores que se adapten al nuevo visitante o turista".

Siguiendo el relevamiento ITH (2020), a nivel nacional, durante el "Aislamiento Social Preventivo y Obligatorio (ASPO)", el 94\% de los hoteles encuestados solicitó ayuda para el pago de salarios, el 53\% realizó suspensiones de personal, el 52\% suspendió inversiones programadas, el $47 \%$ redujo salarios del personal y tomó créditos bancarios, y un $8 \%$ de los hoteles encuestados cerró definitiva y permanentemente su establecimiento. Se destaca que el $35 \%$ de los establecimientos encuestados ya no posee capital de trabajo, el 15\% posee autonomía de trabajo para 1 mes más, el 17\%, para 2 meses, y el $22 \%$, para 3 meses. 
Es preciso indicar que, al igual que en otros países (UNWTO, 2020b), un conjunto de medidas de tipo fiscales, monetarias y de empleo, se diseñaron con el objetivo de auxiliar al sector turístico. Entre estas medidas se encuentra la inclusión del sector turístico en el Programa de Asistencia de Emergencia al Trabajo y la Producción (ATP) del Gobierno Nacional; el Programa de Preventa, que tiene por objetivo fomentar la demanda de turismo interno en la etapa pospandemia; el Plan de Auxilio, Capacitación e Infraestructura para el Turismo (PACIT); el Fondo de Promoción Turística; el congelamiento de tarifas hasta fin de año y exención del corte de servicios para las empresas del sector. De todos modos, más allá de estas disposiciones gubernamentales de apoyo al sector, algunos encuestados manifestaron cierto desacuerdo respecto a la extensión en el tiempo de las medidas restrictivas respecto a la circulación de personas y las actividades turísticas en el país.

\section{CONSIDERACIONES FINALES}

La innovación reconocida como fuente de ventajas competitivas es probable que juegue un papel clave en la recuperación de la industria hotelera tras la pandemia COVID-19. En esta dirección, frente a la incertidumbre respecto a la recuperación de la demanda y las nuevas erogaciones derivadas del cumplimiento de protocolos e inversiones en innovación y digitalización, las prácticas de gestión de ingresos adquieren una renovada utilidad. En particular, el Yield Management (YM), adecuado para los sectores que operan con capacidad relativamente fija, inventario perecedero y posibilidad de vender su servicio anticipadamente, permite al hotel establecer tarifas de forma flexible en un entorno de creciente dinamismo e incertidumbre como el que plantea el escenario vigente.

Partiendo de estas consideraciones, el estudio propuesto se centró en la adopción del YM por parte de los hoteles de 3 y 4 estrellas de la ciudad de Bahía Blanca, Argentina. Esta localidad urbana de tamaño poblacional medio constituye un centro comercial, financiero y de servicios de influencia regional, nodo de transporte y comunicaciones. La ciudad cuenta, además, con una variada oferta de alojamientos. Los turistas se hospedan en los hoteles de 3 y 4 estrellas analizados, principalmente por razones laborales, cuestiones vinculadas a la demanda de servicios de salud y la realización de trámites en los organismos políticoadministrativos de la ciudad.

De forma distintiva respecto a estudios previos, el análisis propuesto abarca dos períodos: la etapa previa inmediata a la crisis de COVID-19 y la etapa de pandemia COVID-19. Se espera que los resultados encontrados respecto a la etapa anterior a la crisis COVID-19 permitan incrementar el conocimiento empírico sobre el grado de difusión del YM y los factores que condicionan su implementación, específicamente, en hoteles que no pertenecen a grandes cadenas internacionales y se encuentran localizados en países de menor grado de desarrollo. La incorporación al análisis de la crisis COVID-19 permite identificar cambios en la adopción o intensificación en el uso del YM como método exclusivo para la fijación de tarifas. También ofrece una caracterización inicial sobre la predisposición de los hoteles a innovar en tiempos de pandemia COVID-19. 
A modo de síntesis, los resultados relevados para la primera etapa (anterior a la crisis COVID-19) relativos al conocimiento y adopción del YM se encuentran en línea con estudios previos sobre innovación y YM en hotelería para países desarrollados (Chávez Miranda y Ruiz Jiménez, 2005; Chávez Miranda et al., 2014; López-Fernández et al., 2009; Abad et al., 2019; Xiaowen et al.; 2013; Talón Ballestero y González -Serrano, 2012 y 2013; Kimes, 2017). En primer lugar, el estudio de caso propuesto da cuenta de la importancia de la pertenencia a una estructura organizativa de mayor complejidad, aun cuando se trate de cadenas de origen y alcance nacional. En segundo lugar, el segmento de la demanda también es un factor que influye en la adopción del YM. Los hoteles que declaran conocer el termino YM y su definición e implementar esta filosofía de gestión son aquellos de mayor categoría (4 y 3 estrellas superior) y pertenecientes a una cadena hotelera. Los hoteles de categoría 3 estrellas y que, coincidentemente no pertenecen a una cadena, no aplican el YM y no cuentan con conocimiento previo sobre el mismo En estos últimos hoteles, la fijación de tarifas se basa en la variación de los costos en función de la inflación y el tipo de cambio nominal.

En tercer lugar, en línea con el estudio de Chávez Miranda y Ruiz Jiménez (2005), todos los entrevistados señalan que usan fuentes de información disponibles (revistas especializadas, Internet) para actualizarse sobre nuevas prácticas de fijación de tarifas. Es preciso destacar que, los hoteles bajo estudio que adoptan el YM también se actualizan a través de una gran variedad de fuentes de información, tales como cámaras empresariales, huéspedes, proveedores, competidores. En cuarto lugar, el surgimiento de numerosos canales de distribución vuelve más complejo el proceso de toma de decisiones sobre fijación de tarifas y requiere de personal calificado y afectado específicamente a tal tarea. Sin embargo, la ausencia de la figura del Yield Manager es un resultado que se deriva del estudio de caso propuesto. Los hoteles de 3 y 4 estrellas superior adquieren tecnología incorporada en softwares específicos al sector, sin embargo, no cuentan con personal dedicado exclusivamente a la gestión de ingresos. Tal como se indicó, este resultado es propio de los hoteles de menor tamaño (Ferguson y Smith, 2014).

Un último resultado para la etapa anterior a la crisis COVID-19 refiere a los limitantes que afrontan los hoteles respecto a la aplicación potencial y la adopción efectiva del YM. Los hoteles que implementan el YM indican sobre la percepción negativa de los huéspedes ante cambios en las tarifas, aspecto ampliamente abordado en la literatura (Kimes, 2002; Choi y Mattila, 2004, entre otros). Por otra parte, los entrevistados reconocen que variables relativas al entorno macro (incertidumbre, inflación, recesión) afectan la potencial aplicación de nuevas prácticas y herramientas. Este tipo de variables también influyen en las decisiones de inversión en innovación en el sector industrial de Argentina (MINCYT, 2015). En sentido contrario, en estudios sobre innovación en hotelería (López-Fernández et al., 2009), y específicamente, sobre el uso del YM (Chávez Miranda y Ruiz Jiménez, 2005 y Chávez Miranda et al., 2014), los factores de contexto macro tienen una influencia moderada respecto a factores internos a la empresa. De este modo, este último resultado marca una diferencia en relación a estudios previos centrados en países de mayor grado de desarrollo.

En relación a la segunda etapa relativa al período actual de pandemia COVID-19, entre los hoteles analizados no se identifican cambios respecto a la adopción y uso del YM. Los hoteles que no implementaban el YM en el período pre-pandemia, no planean su adopción 
en el corto plazo. Los hoteles que aplicaban el YM previamente al COVID-19 reconocen que es una herramienta importante para la fijación de tarifas en el contexto actual, aunque debe combinarse con otros criterios, como los acuerdos de precios a nivel sectorial. Esta conducta se inscribe en una conducta más generalizada respecto a la definición de estrategias de innovación. La información recopilada no ofrece evidencia a favor de la percepción de la crisis como una ventana de oportunidad para invertir en innovación y digitalizar procesos y tareas. Los hoteles analizados, independientemente de su tamaño, categoría y pertenencia a una cadena, coinciden en que la excesiva extensión en el tiempo de las restricciones impuestas por el gobierno nacional respecto a la apertura y reanudación de las actividades turísticas, incluyendo el servicio de alojamiento, es un factor que opera no solo en detrimento del planeamiento de estrategias sino también en detrimento de la propia supervivencia de las firmas. De este modo, al igual que en el período pre-pandemia, se pone en evidencia la importancia de factores externos a los hoteles y específicos al país bajo estudio.

Por último, el análisis propuesto presenta las limitaciones propias del estudio de caso como método de investigación. Si bien el estudio de caso proporciona una generalización de tipo analítica, no es posible derivar generalizaciones estadísticas desde un caso en particular (Yin, 2011). Por otra parte, un aspecto no explorado en este trabajo y que sugiere lineamientos futuros para la investigación refiere a la capacidad de absorción de las firmas hoteleras y su efecto en la definición de estrategias de innovación como respuesta a la crisis COVID-19. La capacidad de absorción, entendida como la capacidad para identificar, asimilar y explorar información externa a la firma, deriva de la capacitación de los recursos humanos (Cohen y Levinthal, 1990). En esta dirección, Fernández et al. (2011) destacan que el turismo va muy por detrás de otros sectores con respecto a la calificación de sus recursos humanos debido a una rotación excesiva, mayor inestabilidad, movilidad, contratos de trabajo de baja calidad. Esto constituye un limitante para el desempeño innovador y económico de las empresas del sector teniendo en cuenta la relevancia del capital humano (Fernández et al., 201 1; Nieves et al., 2014; Plaza Bolaños y Nieves, 2020).

\section{REFERENCIAS}

Abad, Pilar, De la Fuente-Cabrero, C., González-Serrano, Ly., \& Talón-Ballestero, P. (2019). Determinants of successful revenue management. Tourism Review, 74(3), 666-678

Benckendorff, P. Sheldon, P.J., \& Fesenmaier, D.R. (2019). Tourism information technology (3rd Edition) Oxford, UK: CABI.

Boaria, F., \& Gadotti dos Anjos, S.J. (2014). Revenue management a gestão de receitas na hotelaria em foz do Iguaçu - PR. Turismo - Visão e Ação, 16(1),92-114. https://www.redalyc.org/articulo. oa?id=2610/261056066005

Plaza Bolaños, J., \& Nieves, J. (2020). Impacto de las prácticas de recursos humanos en el capital humano y los resultados organizativos de las empresas hoteleras. Cuadernos de Turismo, 45, 311 332. https://doi.org/10.6018/turismo.426151

Cohen, W.M., \& Levinthal, D.A. (1990). Absorptive capacity: A new perspective on learning and innovation. Administrative Science Quarterly, 128-152. DOI: 10.2307/2393553. 
Chávez Miranda, M.E., Ruiz Jiménez, A., \& Perez Diez De Los Rios, J.L. (2014). Factores críticos que dificultan la aplicación de Revenue Management en hoteles urbanos. International Journal of World of Tourism, 1 (1): 26-41.

Chávez Miranda, M.E., \& Ruiz Jiménez, A. (2005). Yield Managerment en Hotelería. Estudio de su aplicación en Sevilla. Ayuntamiento de Sevilla.

Cheraghi, S. H., Dadashzadeh, M., \& Venkitachalam, P. (2010). Revenue management in manufacturing: a research landscape. Journal of Business \& Economics Research (JBER), 8(2). https://doi. org/10.19030/jber.v8i2.673

Choi, S., \& Mattila, A.S. (2004). Hotel revenue management and its impact on customers' perceptions of fairness. Journal of Revenue and Pricing Management, 2(4), 303-314. https://doi.org/10.1057/ palgrave.rpm.5170079

Chicheri, J. (2020). The New Revenue: El libro blanco del Revenue Management, la Distribución y el Marketing Hotelero postCOVID. Revenueknowmads. Disponible en: https://www.revenueknowmads. com/blog/el-libro-blanco-del-revenue-management-la-distribucion-y-el-marketing-hotelero/

Dhar, R.L. (2015). The effects of high performance human resource practices on service innovative behaviour. International Journal of Hospitality Management, 51, 67-75. https://doi.org/10.1016/j. ijhm.2015.09.002

Erdem, M., \& Jiang, L. (2016). An overview of hotel revenue management research and emerging key patterns in the third millennium. Journal of Hospitality and Tourism Technology, 7(3), 300-312. https:// doi.org/10.1108/JHTT-10-2014-0058

Ferguson, M., \& Smith, S. (2014). The changing landscape of hotel revenue management and the role of the hotel revenue manager. Journal of Revenue and Pricing Management, 13(3), 224-232. https://doi.org/10.1057/rpm.2014.11

Fernández, J.I.P, Cala, A.S., \& Figueroa Domeca, C. (2011). Critical external factors behind hotels' investments in innovation and technology in emerging urban destinations. Tourism Economics, 17(2), 339-357. https://doi.org/10.5367/te.2011.0033

Gorenstein, S., Napal, M., Pasciaroni, C., \& Barbero, A. (2012). Bahía Blanca. Una lectura estilizada de su rol y funciones de intermediación. En S. Gorenstein, J. Hernández (comp.): Economía urbana y ciudades intermedias. Trayectorias pampeanas y norpatagónicas. Buenos Aires: Editorial CICUSS

Hao, F., Xiao, Q., \& Chon, K. (2020). COVID-19 and China's hotel industry: Impacts, a disaster management framework, and post-pandemic agenda. International Journal of Hospitality Management, 90, 102636. https://doi.org/10.1016/j.jhm.2020.102636

INDEC - Instituto Nacional de Estadística y Censos de Argentina (2019): Encuesta de Ocupación Hotelera. Informes técnicos, vol. 4, n² 29.

INDEC - Instituto Nacional de Estadística y Censos de Argentina (2020a): Estadísticas de turismo internacional. Septiembre y tercer trimestre de 2020. Informes técnicos, vol. 4, nº 203.

INDEC - Instituto Nacional de Estadística y Censos de Argentina (2020b): Encuesta de ocupación hotelera. Agosto de 2020. Informes técnicos, vol. 4, nº 193. 
INDEC - Instituto Nacional de Estadística y Censos de Argentina (2020c): Encuesta de ocupación hotelera. Diciembre de 2020. Informes técnicos, vol. 5, nº 33.

ITH (2020): Resultados. $2^{\circ}$ Relevamiento del Impacto de la Pandemia por el Covid-19 en los Hoteles. Resumen Ejecutivo. Agosto 2020.

Ivanov, S.(2006). Management of overbookings in the hotel industry-basic concepts and practical challenges. Tourism Today, 6, 19-32.

Ivanov, S. (2014). Hotel revenue management: From theory to practice. Varna: Zangador.

Ivanov, S., \& Ayas, Ç. (2017). Investigation of the revenue management practices of accommodation establishments in Turkey: An exploratory study. Tourism Management Perspectives, 22, 137-149. https://doi.org/10.1016/j.tmp.2017.03.007

Jiang, Y., \& Wen, J. (2020): "Effects of COVID-19 on hotel marketing and management: a perspective article", International Journal of Contemporary Hospitality Management, 32 (8), 2563-2573. https:// doi.org/10.1 108/IJCHM-03-2020-0237

Johnson, E. (2006). Yielding results: removing the human factor from pricing may boost revenue. Journal of Property Management, 71 (1), 42-48.

Jones, P. (2000). Defining yield management and measuring its impact on hotel performance. En A. Ingold et al. (eds): Yield Management Strategies for the Service Industries (pp.85-97). London: Continuum.

Kussmann, M. (2020). Will the coronavirus crisis force hoteliers to implement new technologies faster? Disponible en: https://www.hotel-online.com/press_releases/release/will-the-coronavirus-crisisforce-hoteliers-to-implement-new-technologies-faster/

Kimes, S.E. (1989). The basics of yield management. Cornell Hotel and Restaurant Administration Quarterly, 30(3), 14-19. https://doi.org/10.1177/001088048903000309

Kimes, S.E. (2002). Perceived fairness of yield management. Cornell Hotel and Restaurant. Administration Quarterly, 43(1), 21-30. https://doi.org/10.1177/0010880402431002

Kimes, S.E. (2017). The future of hotel revenue management. Cornell Hospitality Report, 17(1), 3-10.

Kimes, S.E., \& Chase, Richard B. (1998). The strategic levers of yield management. Journal of Service Research, 1 (2), 156-166. https://doi.org/10.1177/109467059800100205

Kimes, S.E., \& Wirtz, J. (2003). Has revenue management become acceptable? Findings from an international study on the perceived fairness of rate fences. Journal of Service Research, 6(2), 125135. https://doi.org/10.1177/1094670503257038

Law, R., \& Jogaratnam, G. (2005).A study of hotel information technology applications. International Journal of Contemporary Hospitality Management, 17(2), 170-180. https://doi. org/10.1108/09596110510582369

López-Fernández, M.C., Serrano-Bedia, A.M. , \& Gómez-López, R. (2009). La decisión de innovar de las empresas turísticas: un análisis empírico de la industria hostelera. Investigaciones Europeas de Dirección y Economía de La Empresa, 15(3), 169-182. https://doi.org/10.1016/\$1 135-2523(12)60107-5 
Lundvall, B. (2010). National Systems of Innovation: Toward a Theory of Innovation and Interactive Learning (Vol. 2). London-New York: Anthem Press.

Martínez-Ros, E., \& Orfila-Sintes, F. (2009). Innovation activity in the hotel industry. Technovation, 29(9), 632-641. https://doi.org/10.1016/j.technovation.2009.02.004

MINCYT- Ministerio de Ciencia, Tecnología e Innovación de Argentina (2015): Encuesta Nacional de Dinámica del Empleo y la Innovación. Resultados Período de cobertura: 2010-2012.

Murimi, M., Billy W. y Olielo, T. (2021). Determinants of revenue management practices and their impacts on the financial performance of hotels in Kenya: a proposed theoretical framework. Future Business Journal, 7(1), 1-7. https://doi.org/10.1186/s43093-020-00050-9

Nieves, J., \& Diaz-Meneses, G. (2018). Knowledge sources and innovation in the hotel industry. International Journal of Contemporary Hospitality Management, 30(6), 2537-2561. https://doi. org/10.1 108/IJCHM-07-2016-0341

Nieves, J., \& Osorio, J. (2019). Using information technology to achieve management innovation. Academia Revista Latinoamericana de Administración, 32 (1): 20-39. https://doi.org/10.1 108/ARLA02-2016-0037

Nieves, J., Quintana, A., \& Osorio, J. (2014).Knowledge-based resources and innovation in the hotel industry. International Journal of Hospitality Management, 38, 65-73. https://doi.org/10.1016/j. ijhm.2014.01.001

Nieves, J., \& Segarra-Ciprés, M. (2015). Management innovation in the hotel industry. Tourism Management, 46, 51-58. https://doi.org/10.1016/j.tourman.2014.06.002

OECD (2020). Tourism Policy Responses to the coronavirus (COVID-19). Disponible en: http://www.oecd. org/coronavirus/policy-responses/rebuilding-tourism-for-the-future-covid-19-policy-responses-andrecovery-bced9859/

O' Connor, P., \& Murphy, J. (2008). Hotel yield management practices across multiple electronic distribution channels. Information Technology \& Tourism, 10(2), 161-172. https://doi. org/10.3727/109830508784913103

Okumus, F. (2004). Implementation of yield management practices in service organisations: empirical findings from a major hotel group. The Service Industries Journal, 24(6), 65-89. https://doi. org/10.1080/0264206042000299185

Orfila-Sintes, F., \& Mattsson, J. (2003). Determinantes de los tipos de innovación tecnológica en la actividad hotelera, en XII Simposio Internacional de Turismo y Ocio. Univsersitat de les Illes Balears.

Orfila-Sintes, F., \& Mattsson, J. (2009). Innovation behavior in the hotel industry. Omega, 37(2), 380-394. https://doi.org/10.1016/j.omega.2007.04.002

Ottenbacher, M., \& Harrington, R. (2010).Strategies for achieving success forinnovative versus incremental new services. Journal of Services Marketing, 24(1), 3-15. https://doi.org/10.1 108/08876041011017853

Pinassi, C.A., \& Ercolani, P. (2012). El turismo urbano-metropolitano y su configuración espacial en las ciudades. Análisis de la focalidad urbana en Bahía Blanca (Argentina). Revista TURyDES, 5(13). 
Schroeder, R., \& Formiga, N. (2009). Las actividades turístico-recreativas como dinamizadoras de centros urbanos pequeños y áreas rurales. El caso del área de influencia de Bahía Blanca. Estudios territoriales. Departamento de Geografía y Turismo, Universidad Nacional del Sur.

Shin, H., \& Kang, J. (2020). Reducing perceived health risk to attract hotel customers in the COVID-19 pandemic era: Focused on technology innovation for social distancing and cleanliness. International Journal of Hospitality Management, 91, 102664. https://doi.org/10.1016/j.jjhm.2020.102664

Sigala, M. (2003). The information and communication technologies productivity impact on the UK hotel sector. International Journal of Operations \& Production Management, 23(10), 1224-1245. https://doi.org/10.1108/01443570310496643

Smith, B.C., Leimkuhler, J.F., \& Darrow, Ross M. (1992). Yield management at American Airlines. Interfaces, 22(1), 8-31. https://doi.org/10.1287/inte.22.1.8

Talón Ballestero, P., Figueroa Domecq, C., \& Vacas Guerrero, C. (2008). Estrategias de implantación del yield management: el caso de la hotelería madrilena. En: Estableciendo puentes en una economía global (p. 22), España, Escuela Superior de Gestión Comercial y Marketing, ESIC.

Talon Ballestero, P., \& González-Serrano, L. (2012). Yield Revenue Management in the Hotel Sector: An Empirical Analysis of Its Application and Results in Madrid, Spain. Quantitative Methods in Tourism Economics, 213-231. https://doi.org/10.1007/978-3-7908-2879-5_12

Talon Ballestero, P., \& González-Serrano, L. (2013). Future trends in revenue management. Journal of Revenue and Pricing Management, 12 (3): 289-291 https://doi.org/10.1057/rpm.2012.50

UNWTO (2020a). World Tourism Barometer May 2020 Special focus on the Impact of COVID-19.

UNWTO (2020b). How are countries supporting tourism recovery? UNWTO Briefing Note - Tourism and Covid-19, Issue 2 June 2020.

Xiaowen, W., Xin, T., Kai, L., \& Zhijian, H. (2013). Research on factors influencing hotel revenue management decision-making and performance: an empirical study based on high star-rated hotels in China. Tourism Tribune/Lvyou Xuekan, 28(9), 25-35.

Yin, R.K. (201 1). Applications of case study research-3rd Ed. London: SAGE.

Contribución de cada autor:

Carolina Pasciaroni: concepción, revisión de la literatura, estructuración del marco teórico, trabajo de campo, análisis de datos, discusión de resultados, conclusiones y redacción y revisión final.

Silvina Elías: concepción, revisión de la literatura, estructuración del marco teórico, discusión de resultados, conclusiones y revisión final.

José Ignacio Diez: discusión de resultados y revisión final. 